Franz Overbeck

Werke und Nachlaß 5

Kirchenlexicon Texte

Ausgewählte Artikel J-Z 


\title{
Franz Overbeck Werke und Nachlaß
}

\author{
Editionskommission
}

Prof. Dr. theol. Ekkehard W. Stegemann (Basel), Präsident

Prof. Dr. theol. Rudolf Brändle (Basel)

Prof. Dr. phil. Hubert Cancik (Tübingen)

Dr. Hildegard Cancik-Lindemaier (Tübingen)

Dr. phil. Bernd Lutz (Stuttgart)

Prof. Dr. phil. Karl Pestalozzi (Basel)

Dr. theol. Niklaus Peter (Basel)

Dr. phil. Barbara von Reibnitz (Basel/Berlin)

Prof. Dr. theol. Martin Anton Schmidt (Basel)

Dr. phil. Mathias Stauffacher (Basel)

Marianne Stauffacher-Schaub (Basel)

Verlag J.B. Metzler

Stuttgart $\cdot$ Weimar 


\title{
Franz Overbeck Werke und Nachlaß Kirchenlexicon Texte Ausgewählte Artikel J-Z
}

\author{
In Zusammenarbeit mit \\ Marianne Stauffacher-Schaub \\ herausgegeben von \\ Barbara von Reibnitz
}

Verlag J.B. Metzler

Stuttgart · Weimar 


\section{Publiziert mit Unterstützung des}

Schweizerischen Nationalfonds zur Förderung der wissenschaftlichen Forschung

Die Deutsche Bibliothek - CIP-Einheitsaufnahme

\section{Overbeck, Franz:}

Werke und Nachlaß / Franz Overbeck. Ed.-Komm.: Ekkehard

W. Stegemann ...- Stuttgart : Metzler.

ISBN 978-3-476-01210-4

NE: Stegemann, Ekkehard W. [Hrsg.]; Overbeck, Franz: [Sammlung]

5. Kirchenlexicon : Texte, - ausgewählte Artikel J-Z / in Zusammenarbeit mit

Marianne Stauffacher-Schaub hrsg. von Barbara von Reibnitz. - 1995

ISBN 978-3-476-00966-1 ISBN 978-3-476-98747-1 (eBook)

DOI $10.1007 / 978-3-476-98747-1$

\section{ISBN 978-3-476-01210-4 (Gesamtwerk) \\ ISBN 978-3-476-00966-1 (Band 5)}

Dieses Werk einschließlich aller seiner Teile ist urheberrechtlich geschützt. Jede Verwertung außerhalb der engen Grenzen des Urheberrechtsgesetzes ist ohne Zustimmung des Verlages unzulässig und strafbar. Das gilt insbesondere für Vervielfältigungen, Übersetzungen, Mikroverfilmungen und die Einspeicherung und Verarbeitung in elektronischen Systemen.

C Springer-Verlag GmbH Deutschland 1995

Ursprünglich erschienen bei J. B. Metzlersche Verlagsbuchhandlung und Carl Ernst Poeschel Verlag GmbH in Stuttgart 1995 
Inhaltsübersicht

Editorische Legende . . . . . . . . . . . . . . . VII

Artikelverzeichnis . . . . . . . . . . . . . . . . . IX

Texte . . . . . . . . . . . . . . . . . 1

Abkürzungen . . . . . . . . . . . . . . . . . . . . 695

Verzeichnis der von Franz Overbeck selbst publizierten Schriften 697

Verzeichnis der Publikationen aus Franz Overbecks Nachlass . 699

Bibliographie der von Franz Overbeck zitierten Literatur . . . 700

Ausgaben . . . . . . . . . . . . . . . . . . . . 700

Literatur . . . . . . . . . . . . . . . . . 702

Register . . . . . . . . . . . . . . 734 


\section{Editorische Legende}

Vgl. Einleitung und Editorische Notiz in Bd. 4.

Im Text und im textkritischen Apparat verwendete editorische Zeichen: 〈text〉 Ergänzungsvorschlag der Herausgeberin

[text] Tilgungsvorschlag der Herausgeberin

\{text $\quad$ Autorstreichung

$\left\ulcorner_{\text {text }}\right\urcorner \quad$ Autoreinfügung

$[\mathrm{x}\} \quad$ Überschreibung einzelner oder mehrerer Buchstaben

${ }^{+}$text nicht sicher entzifferter Text

Sonstige in Text und Apparat verwendete Zeichen und Abkürzungen:

| Seitenwechsel im Manuskript

a Verweis auf den textkritischen Apparat

- in dieser Auswahl gedruckter Artikel

$\langle C u K\rangle \quad$ der Artikel wurde ganz oder teilweise in der Kompilation »Christentum und Kultur« verwendet

I frühe Hand (Leipzig bis Basel: Studienzeit und erste Lehrtätigkeit, ca. 1856-1870)

II mittlere Hand (Basel: Lehrtätigkeit bis zur Emeritierung, ca. 1870-1897)

III späte Hand (Basel: nach der Emeritierung, ca. 1897-1905)

dat. O.: Datierung Overbecks

L: $\quad$ Erscheinungsdatum der von Overbeck zitierten Literatur

\section{Autorabkürzungen:}

Die von Overbeck verwendeten Abkürzungen lassen sich weitgehend systematisieren nach:

a) Endabkürzungen (Wegfall der Endsilbe)

- histor. historisch

- freil. freilich

- ebendas. ebendaselbst

- besond. besonders

- unt. unter 
b) Binnenabkürzungen (Wegfall eines oder mehrerer Binnenvokale, z. Tl. mit anschliessendem Konsonanten), etwa:

- ht $/$ kt heit $/$ keit; z. B. Krankht $=$ Krankheit, Christlichkt $=$ Christlichkeit

- g ung/üng/ang; z. B. Behandlg = Behandlung

$-\mathrm{d} \quad$ und/and/end; z. B. Bewdtniss $=$ Bewandtniss

- k unk, ank; z. B. pkt = punkt

- Wegfall von ie, eu, au etc.; z. B. Brf $/$ Bf = Brief, behptet $=$ behauptet

Diese Abkürzungen treten häufig kombiniert auf:

- ursprgli. ursprünglich

- wahrschl. wahrscheinlich

- Bdtg Bedeutung

- Behdlg Behandlung

Daneben benutzt Overbeck häufig die folgenden Abkürzungen:

- Xus $\quad$ Christus $(\mathrm{X}=$ griech. chi)

- Xsten Christen

- Xsthm Christenthum

- K Kirche

- Kg./KG. Kirchengeschichte

- KVV. Kirchenväter

- WW. Werke, Worte

- BB. Bücher

- Ss. Seiten 


\section{Artikelverzeichnis}

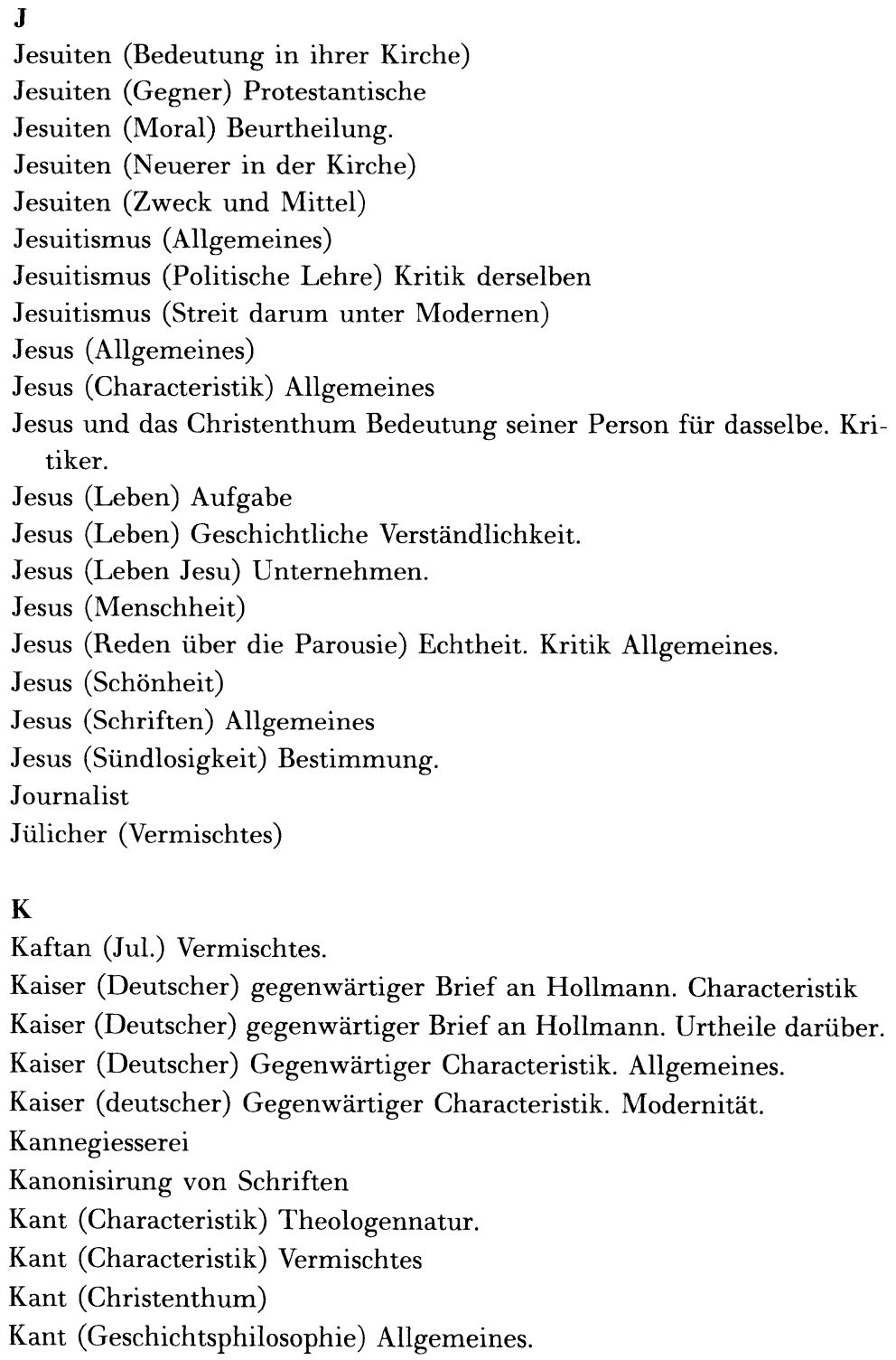


Kant u. Protestantismus.

Kant $u$. die Religion Allgemeines.

Katholicismus (moderner) Allgemeines.

Katholicismus und Protestantismus Gegensatz

Katholicismus und Protestantismus. Histori. Betrachtung des Gegensat zes.

Katholicismus (Weltlichkeit)

Kierkegaard (Vermischtes)

Kirche (Abendland und Morgenland)

Kirche u. Absolutismus.

Kirche (Allgemeinheit) Allgemeines.

Kirche und Alterthum Allgemeines.

Kirche und Christenthum Unterschied.

Kirche (Definition)

Kirche und Democratie

Kirche (Mittelalter) Allgemeines.

Kirche u. Staat (Trennung) America

Kirche und Staat (Trennung) Gründe.

Kirche und Staat. (Vermischtes)

Kirchengeschichte (Profane)

Kirchengeschichte und Theologie

Kirchengeschichte und Weltgeschichte. Beziehungen zwischen ihnen

Kirchengeschichtschreibung (Anfänge) Allgemeines.

Kirchenregiment.

Kirchenverfassung (Allgemeines)

Kritik (Allgemeines)

Kritik und Schwärmerei

Künstler (Allgemeines)

L

Lagarde (P. A. de) Characteristik Allgemeines

Lagarde (P. A. de) Historiker.

Lagarde (P. A. de) Religion.

Langbehn

Liberalismus (Vermischtes)

Lichtenberg, G.Chr. (Allgemeines)

Litteratur (christliche) Allgemeines.

Litteratur (christliche) Characteristisches. Scheu vor sich.

Litteratur (christliche) Entstehung. Vermischtes. 
Litteratur (christliche) 4. Jahrhundert.

Litteratur u. Cultur.

Litteratur u. Juden

Litteratur (religiöse) Allgemeines

Litteratur (Zukunft)

Litteraturgeschichte (Allgemeines)

Litteraturzeitung (Theologische) Vermischtes

Loisy (A.) Litteratur.

Loisy (A). Vermischtes.

Lucretius (Vermischtes)

Luther und Denifle.

Luther und Erasmus.

Luther (Gegenwart) Vermischtes.

Luther (Lehre) Glaubensbegriff. Allgemeines.

Luther $u$. Mittelalter.

Luther $u$. Nietzsche

Luther (Uebermensch)

Luther (Urtheile über ihn) Neuere. Gegner.

M

Macht (Allgemeines)

Mann und Weib. (Allgemeines)

Maurenbrecher Religionsproblem.

Meinung (öffentliche) Vermischtes

Memento mori des Christenthums

Menschenverstand (gesunder)

Mission (Allgemeines).

Mission (christliche) Protestantische. Allgemeines.

Mittelalter (Allgemeines)

Modernismus und Modernität.

Modernität (Allgemeines)

Modernität (Gegenwart) Allgemeines.

Modernität (meine)

Modernus (Allgemeines)

Modernus. (Vermischtes)

Mommsen (Th.) Litteratur.

Monatsschrift für die kirchliche Praxis. (Characteristik)

Monotheismus des Christenthums. Allgemeines.

Moral (Allgemeines) 
Moral u. Beruf Gegenwart.

Moral und Ethik

Moral u. Moralität. Allgemeines

Moralität (Vermischtes)

Mythus

$\mathbf{N}$

Napoleon (Allgemeines)

Napoleon und das Christenthum.

Napoleon u. Religion.

Nationalismus (Allgemeines)

Nationalismus (Deutscher) Characteristisches.

Nationalismus (Gegenwart) Characteristisches.

Nationalismus (Gegenwart) Christenthum.

Nationalismus (Gegenwart) Deutscher.

Natur u. Geist

Naturforschung (Gegenwart) Bildender Werth.

Naumann (Fr.) Vermischtes.

Die Artikel "Nietzsche ..." in OWN 7

O

Objectivität (Vermischtes)

Offenbarung als Erziehung des Menschengeschlechts.

Orthodoxie

$\mathbf{P}$

Papyrusphilologie (neueste) Vermischtes.

Pascal Apologie des Christenthums Characteristik. Allgemeines.

Pascal u. der Jansenismus

Pascal Jesuiten

Pascal Katholicismus

Pascal u. die Kirche

Pascal u. Paulus.

Pascal Provinciales Kritik ihrer Kritik

Paulus

Paulus. Allgemeines

Paulus (Apostolat)

Paulus (Briefe überhaupt). Reihenfolge im Canon. 
Paulus (Characteristik) Apostolische Wirksamkeit. Eile.

Paulus (Characteristik) Apostolische Wirksamkeit. Strenge.

Paulus (Characteristik) Fanatismus.

Paulus u. Jesus (Allgemeines)

Paulus $u$. das Judenthum

Paulus (Lehrbegriff) Gesetz. Allgemeines.

Paulus u. die Parousie.

Paulus Reformatorische Auffassung

Paulus Selbstbewusstsein

Paulus (Selbständigkt) Ob Stifter des Christenthums?

Paulus (Universalismus)

Paulus und die Urapostel

Pessimismus (Allgemeines)

Pessimismus und Optimismus.

Pessimismus. (Vermischtes)

Pfleiderer (Otto) als moderner Theologe.

Phantasie (Allgemeines)

Philisterthum

Philologie (Allgemeines)

Philologie (Theologie). Gegenwart.

Philosophie (Gegenwart) Vermischtes.

Philosophie und Geschichte. Vermischtes.

Philosophie u. Leben

Philosophie u. Schwärmerei

Pietismus (Allgemeines)

Pietismus und Aufklärung Vermischtes.

Pietismus und Orthodoxie Gegenwart.

Presse (moderne) Allgemeines.

Preussen $u$. das Christenthum

Protestantismus $u$. Katholicismus Gegensatz. Allgemeines.

Protestantismus u. Staat.

Protestantismus (Vermischtes)

Proudhon (Religion)

Proudhon (Vermischtes)

Q

Quellenanalyse (Allgemeines) 
$\mathbf{R}$

Radicalismus (Allgemeines)

Rationalismus (Allgemeines)

Rationalismus (Wunderkritik)

Rationalismus des 18. Jahrhunderts. Allgemeines.

Realismus moderner (Allgemeines)

Reformation (Allgemeines)

Reformation (moderne Auffassg)

Reformation und Renaissance Allgemeines.

Religion (Allgemeines)

Religion $u$. Beruf (Allgemeines)

Religion (Ende)

Religion (Entbehrlichkeit)

Religion (Entstehung) Vermischtes.

Religion (Gegenwart)

Religion (Gegenwart) Apologie

Religion (Gegenwart). Vermischtes

Religion und Germanen. Allgemeines.

Religion (Geschichte).

Religion (Humanität).

Religion und Kritik (Allgemeines)

Religion und Kunst (Allgemeines)

Religion (meine)

Religion und Moral. Gegensatz

Religion und Moral. Neuere Theologen.

Religion und Moral Vermischtes.

Religion und Mythus

Religion (Nutzen)

Religion und Offenbarung

Religion Orient $u$. Occident Allgemeines.

Religion (Popularität)

Religion (Rationalismus) Allgemeines

Religion (Schaden)

Religion und Staat.

Religion (Stifter)

Religion und Tradition

Religion (Ueberflüssigkeit)

Religion (Verfall)

Religion und Wissenschaft. Allgemeines 
Religionsgeschichte (Allgemeines)

Religionsgeschichte (Gegenwart) Allgemeines.

Religionsgeschichte und Theologie. Allgemeines.

Religionsgeschichte (vergleichende) Allgemeines.

Religionspolitik (moderne) christliche. Allgemeines.

Religionswissenschaft (Gegenwart) Allgemeines.

Rembrandtdeutscher.

Republik und Kirche.

Rhetorik (Gefahren)

Ritschl als Apologet.

Ritschl u. Baur.

Ritschl (Biographie des Sohnes)

Ritschl und Bossuet.

Ritschl (Characteristik) Geselligkeit

Ritschl Characteristik Humor.

Ritschl Characteristik Jovialität.

Ritschl (Charakteristik) Männlichkeit

Ritschl (Characteristik) Meister der Theologie.

Ritschl (Characteristik) Optimismus.

Ritschl (Characteristik) Popularität.

Ritschl (Characteristik) Reformator.

Ritschl (Characteristik) Theolog.

Ritschl (Characteristik) Vermischtes.

Ritschl u. Doellinger

Ritschl (Dogmatiker)

Ritschl als Historiker

Ritschl u. Kirchengeschichte

Ritschl Lehre Gottheit Christi.

Ritschl u. Lipsius.

Ritschl u. Luther.

Ritschl Metaphysik und Theologie Absage.

Ritschl und Pascal.

Ritschl und der Pietismus. Gegensatz

Ritschl u. Schleiermacher

Ritschl Theologie Allgemeines.

Ritschl u. Tübinger Schule

Ritschl (Vater)

Ritschl mein Verhältniss zu ihm.

Rocholl Einsame Wege Vermischtes 
Die Artikel »Rohde ... in OWN 7

Rohrbach Im Lande Jahwe's Vermischtes.

Romantik (Allgemeines)

Rothe (Characteristik) Allgemeines.

Rothe Characteristik Separatismus.

Rothe u. Pietismus.

Rothe System Kritik desselben

S

Schleiermacher (Allgemeines)

Schleiermacher (Christologie)

Schleiermacher u. Spinoza.

Schopenhauer (Professoren der Philosophie)

Schrift und Wort.

Schriftglaube (moderner)

Schriftstellerei bei den Alten.

Schriftstellerei und Bibel

Schriftstellerei und Christenthum

Schriftstellerei (Gegenwart) Motive

Schriftstellerei (Gegenwart) Vermischtes.

Schriftstellerei (kirchliche) Anfänge. Vermischtes.

Schriftstellerei (kirchliche) Exegetische. Allgemeines.

Schriftstellerei (kirchliche) Umfang.

Schriftstellerei (kirchliche) Vermischtes.

Schriftstellerei (kirchliche) u. Weltlitteratur.

Schuldgefühl $u$. Religion

Schweigen.

Seeberg (R.) Schriften.

Seelenwanderung (Vermischtes)

Selbstgefälligkeit.

Selbstkenntniss.

Selbstmord (Christenthum)

Selbstmord (Neuere Zeit)

Selbstmord (Vermischtes)

Sinn des Lebens. Allgemeines.

Socialismus (Allgemeines)

Socialismus u. Anarchismus. Vergleichung.

Socialismus und Christenthum Gegenwart. 
Socialismus (nationaler)

Socialismus und Religion Allgemeines.

Sorgenvolle (Der)

Spitteler (Carl)

Sprache (Allgemeines)

Sprachvergleichung (Allgemeines)

Staat (Allgemeines)

Staat $u$. Reformation.

Staat (Zweck)

Sterne (Lorenz)

Stil (mein)

Stirner (Max) Allgemeines

T

Taine (H.) Characteristik

Taine (Hipp.) u. Christenthum.

Taine u. Guizot.

Taine (Hipp.) Kritik. Allgemeines.

Teufel (Vermischtes)

Theologen (Dummheit)

Theologen (moderne) Figaro's

Theologen (Moral)

Theologen (philosophische)

Theologie (Allgemeines)

Theologie (Aufgabe).

Theologie (Characteristik) Humanität.

Theologie (Characteristi. aus der neueren)

Theologie (christliche) Geschichte Anfänge

Theologie (Christlichkeit) Bestreitung.

Theologie (Einheit)

Theologie (Gegenwart) Kritik des Xsthms.

Theologie im Heidenthum.

Theologie (meine)

Theologie und Metaphysik. (Vermischtes).

Theologie (moderne) Accomodation.

Theologie (moderne) Anfänge. Vermischtes.

Theologie (moderne) Arbeitstheilung

Theologie (moderne) Bunsen.

Theologie (moderne) Characteristik. Modernität. 
Theologie (moderne) Christus.

Theologie (moderne) Cultur.

Theologie (moderne) Deutsches Reich. Allgemeines

Theologie (moderne) Geschichte derselben.

Theologie (moderne) Geschichtswissenschaft Allgemeines.

Theologie (moderne) u. Ich.

Theologie (moderne) Jugend

Theologie (moderne) Litteratenhaftigkeit.

Theologie (moderne) Mystik.

Theologie (moderne) Offenbarungsbegriff.

Theologie (moderne) Philologie

Theologie (moderne) Politik.

Theologie (moderne) Reformation (Auffassung derselben)

Theologie (moderne) Religionsgeschichte. Allgemeines.

Theologie (moderne) Rothe

Theologie (moderne) Tübinger Schule.

Theologie (moderne) Urchristenthum.

Theologie (moderne) Vor- und Nebengeschichte

Theologie (moderne) Vulgärrationalismus.

Theologie (moderne) u. Wagner.

Theologie (moderne) Werth, practischer.

Theologie (Negativität)

Theologie und Philologie

Theologie Rationalismus.

Theologie und Religion. Allgemeines.

Theologie $u$. Religionsgeschichte. Allgemeines

Theologie (Schwärmerei)

Theologie (Vermischtes)

Theologie als Wissenschaft Allgemeines.

Theologie $u$. Wissenschaft Aneignung.

Theologie (Wissenschaft) Exegese. Allgemeines.

Theologie als Wissenschaft. Subjectivität.

Tod (Allgemeines)

Tod u. Christenthum

Tod und Cultur.

Tod (Vermischtes)

Todtschweigen

Toleranz. (Allgemeines)

Traurigkeit und Freude 
Die Artikel »Treitschke... in $O W N 7$

Tübinger Schule u. Moderne Theologie

$\mathbf{U}$

Unsterblichkeit (Individuelle) Begründung

November 1897.

Urchristenthum (Allgemeines)

Urchristenthum (Katholische Auffassung.)

Urchristenthum (Theologische Auffassung).

Urgeschichte (Allgemeines)

Urgeschichte (Gegenwart).

Urgeschichte Tradition Allgemeines.

Urlitteratur (christliche) Begriff. Allgemeines.

Urlitteratur (christliche) Vermischtes.

Usener (Herm.) als Theologe

W

Wagner (Richard) u. Christenthum Vermischtes.

Wagner (Rich) u. Professoren

Wagner (Richard) Vermischtes.

Weiss (Johannes)

Weisse (Chr. H.) Evangelische Geschichte. Kritik des Werks.

Weizsäcker (C.) Apostolisches Zeitalter. Characteristik

Weizsäcker (Carl) Characteristik.

Welt (Allgemeines)

Welt u. Christenthum

Weltkenntniss (moderne).

Welträthsel.

Wernle (Paul) u. Ritschl

Wernle (Paul) Schriften. Allgemeines.

Wernle (Paul) Schriften Urchristenthum

Wilamowitz-Möllendorff Characteristisches.

Wissenschaft (Allgemeines)

Wissenschaft (christliche) Allgemeines

Wissenschaft (Gegenwart) Allgemeines.

Wissenschaft (Gegenwart) Vermischtes

Wissenschaft und Kirche

Wissenschaft $u$. Mensch 
Wissenschaft und Methoden

Wissenschaft (Secularisation) Vermischtes.

Wissenschaft Voraussetzungslosigkt. Vermischtes.

Witz (geistlicher)

Wunderkritik (Vertheidigung)

$\mathbf{Z}$

Zahn (Theod.) Characteristisches.

Zahn und Harnack

Zahn (Th) Tatian

Zeit (Allgemeines)

Zweifel (Allgemeines)

\section{Anhang}

Matthaeusevangelium (Verfasser) Allgemeines.

Matthaeus 5,3. (Patristisches).

Matthaeus 5,5.

Matthaeus 5,9.

Matthaeus 5,39. (Patristisches).

Matthaeus 5,39-41. Patristisches.

Matthaeus 5,44.

Matthaeus 6,3.

Matthaeus 18,3.

Lucasevangelium (Characteristik) Historicismus.

Lucasevangelium (Characteristik) Stil.

Lucasevangelium (Verfasser) Selbsteinführg

Lucas 1, 1-4 Vermischtes.

Lucas 17,20. 21. 